\title{
Klinisches Risikomanagement - Stellenwert im betrieblichen Gesamt-Risikomanagement
}

\author{
Es ist entscheidend, dass in Gesundheitsbetrieben die Unterschiede zwischen \\ gesamtbetrieblichem Risikomanagement (IKS) und klinischem Risikomanagement \\ bewusst berücksichtigt werden. Die speziellen methodischen, organisatorischen \\ und inhaltlichen Anforderungen an das klinische Risikomanagement müssen be- \\ rücksichtigt und zur Führungsaufgabe erklärt werden.
}

\section{Helmut Paula ${ }^{a}$, \\ Marc-Anton Hochreutener ${ }^{b}$, \\ David Schwappach, \\ Dieter Conen, \\ Andreas Toblere}

a Verantwortlicher klinisches Risikomanagement Inselspital, Universitätsspital Bern

b Dr. med., Geschäftsführer Stiftung für Patientensicherheit

c Prof. Dr., wissenschaftlicher Leiter Stiftung für Patientensicherheit

d Prof. Dr. med. Präsident Stiftung für Patientensicherheit

e Prof. Dr. med., ärztlicher Direktor Inselspital, Universitätsspital Bern
Korrespondenz:

Helmut Paula

Inselspital

Universitätsspital Bern

Murtenstrasse 21

CH-3010 Bern

Tel. 0316320571

Fax 0316329615

helmut.paula[at]insel.ch
Das institutionalisierte klinische Risikomanagement ist eine vergleichsweise junge Einrichtung in den Spitälern. Es umfasst die Gesamtheit aller Strukturen, Prozesse, Instrumente und Aktivitäten, welche die Mitarbeitenden eines Spitals dabei unterstützen, die Risiken der Patientenversorgung zu erkennen, $\mathrm{zu}$ reduzieren und zu beherrschen [1]. Es setzt das Erkennen von Fehlern, Beinaheschäden und Schäden voraus und verlangt deren Analyse [2].

Die Ziele des klinischen Risikomanagements sind einerseits die Prävention und Reduktion von Fehlern in der Patientenbehandlung und -betreuung (Primärprävention) und andererseits die Schadensbegrenzung nach dem Auftreten von Fehlern (Sekundärprävention). Es befasst sich also mit den spezifischen Risiken, die erst dadurch entstehen, dass Patienten klinisch behandelt und betreut werden. Es war zwar schon immer eine wichtige Grundlage medizinischen und pflegerischen Handelns, die anvertrauten Patienten vor Schädigungen durch die Behandlung zu bewahren. Allerdings wurde dies über lange Zeit hinweg vor allem als rein fachliche Aufgabe der Disziplinen und Berufsgruppen angesehen. Übergeordnete Sichtweisen mit gezielter Berücksichtigung von Systemfaktoren, Einbeziehung der «Human Factors» und daraus resultierende Massnahmen auf Ebene der

\section{Abbildung 1}

Das gesamtbetriebliche Risikomanagementsystem in Gesundheitseinrichtungen setzt sich meist aus verschiedenen Verantwortlichkeiten, Organisationsbereichen und Risikoaspekten zusammen.

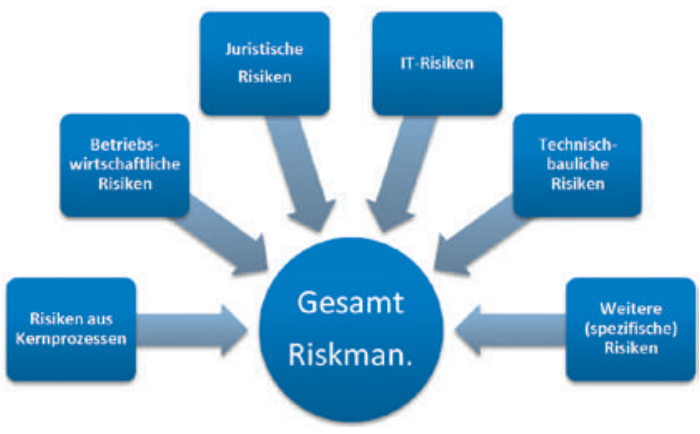

Gesamtorganisation haben sich dagegen erst in den letzten Jahren durchgesetzt. Inzwischen kann man jedoch davon ausgehen, dass in einem grossen Teil der Schweizer Spitäler zumindest Basismassnahmen etabliert sind und im Laufe der Zeit weiter ausgebaut werden. Angesichts der noch relativ kurzen Existenz des klinischen Risikomanagements ist es aber wichtig, seine Rolle und Verankerung in der Organisation zu hinterfragen und klärend zu definieren.

Ein wesentlicher aktueller Aspekt ist dabei die Positionierung des klinischen Risikomanagements im Gesamt-Risikomanagement eines Betriebes: In welcher Beziehung steht klinisches Risikomanagement zum gesamtbetrieblichen Risikomanagement einerseits und zu den klinischen Kernprozessen (Behandlung und Betreuung von Patienten) andererseits? Diese Fragen werden derzeit in vielen Einrichtungen diskutiert und unterschiedlich gelöst. Die Antworten darauf werden die Zukunft des klinischen Risikomanagements massgeblich beeinflussen.

Wie auch bei anderen Unternehmen setzt sich das gesamtbetriebliche Risikomanagementsystem in den Gesundheitseinrichtungen normalerweise aus den verschiedensten Verantwortlichkeiten, Organisationsbereichen und Risikoaspekten zusammen. Dabei werden neben den Gefahren, die sich direkt durch den Kernauftrag ergeben, u.a. auch finanziell-betriebswirtschaftliche, juristische, technisch-bauliche und IT-Risiken behandelt (Abb. 1). Als Grundlage für das gesamtbetriebliche Risikomanagement wird meist das revidierte Obligationenrecht genannt, das die unternehmerische Verpflichtung regelt, sich mit den betrieblichen Risiken auseinanderzusetzen. Zentrales Element ist dabei das sogenannte Interne Kontrollsystem (IKS), das dem Unternehmen helfen soll, Massnahmen und Kontrollen zur Abwehr von Schäden und zur Einhaltung von gesetzlichen Pflichten gezielt zu steuern. Dabei ist allerdings zu beachten, dass das IKS definitionsgemäss nur Risiken behandelt, die sich gefährdend auf das Unternehmen auswirken. Zweifellos gehören dazu auch Patientenschädigungen. Sie können zu höheren Behandlungskosten und Versicherungsprämien, Haftungsansprüchen, Reputa- 
tionsverlusten, Patientenunzufriedenheit und mittelfristig auch zu sinkenden Fallzahlen führen. Folglich sind sie auch als schwerwiegende unternehmerische Risiken zu betrachten.

Das klinische Riskmanagement befasst sich demgegenüber hauptsächlich mit den Kernprozessen eines Spitals und ist deshalb als wichtiger (eigentlich wichtigster) Bestandteil des unternehmensweiten Risikomanagements zu sehen. Es ist notwendig und begrüssenswert, wenn Spitalleitungen und Verwaltungsräte die Thematik entsprechend ernst nehmen und den klinischen Risiken die erforderliche Beachtung schenken. Die Verantwortung eines Spitals, Schaden von seinen Patienten abzuwenden, ergibt
Als ein Hauptgrund für die zu langsamen Fortschritte in der Patientensicherheit wurde national und international die mangelnde Integration von Risikomanagement und klinischer Praxis erkannt. Engagement für Patientensicherheit wird noch von zu vielen Fachpersonen häufig als etwas «Externes», etwas «Zusätzliches», etwas «technokratisch von oben Verordnetes» verstanden. Also als etwas, das nicht Kernbestandteil der klinischen Prozesse ist. Jede organisationelle Struktur, die diesen Eindruck verstärkt und zusätzliche Distanz zwischen Klinik und Risikomanagement schafft, stellt deshalb für die Weiterentwicklung der Patientensicherheit eine ernste Schwächung dar.

\section{Ziele des klinischen Risikomanagements: Prävention und Reduktion von Fehlern sowie Schadensbegrenzung nach dem Auftreten von Fehlern.}

sich allerdings nicht primär aus unternehmerischen oder juristischen Gesichtspunkten, sondern in erster Linie aus der klinischen und aus einer ethisch-moralischen Verpflichtung heraus. Die Tatsache, dass dadurch auch unternehmerische Risiken reduziert werden, ist ein positiver Nebeneffekt, sollte aber nicht die Hauptmotivation für das klinische Risikomanagement sein - und ist es in der Regel hoffentlich auch nicht. Alleine schon aufgrund der unterschiedlichen Grundorientierung und Zielsetzung kann das klinische Risikomanagement deshalb nicht einfach als konventionelles Instrument oder Element des IKS betrachtet werden. Vielmehr sprechen eine ganze Reihe von Gründen dafür, dass das klinische Risikomanagement innerhalb des Gesamt-Risikomanagementsystems eine differenzierte und prominente Sonderstellung einnimmt.

So tragen beispielsweise in den Spitälern die einzelnen Kliniken zu weiten Teilen die Verantwortung für die Patientensicherheit im Rahmen ihrer fachlichen Verantwortung und Therapiefreiheit. IKS beruht hingegen zu weiten Teilen auf einem strikten Top-Down-Ansatz mit verpflichtenden Weisungen und Kontrollen. Unbestritten ist dieser Ansatz auch in Teilaspekten des klinischen Risikomanagements durchaus sinnvoll. Allerdings zeigt die Erfahrung, dass von oben verordnete Massnahmen in der klinischen Praxis nicht ausreichend umgesetzt werden, wenn auf der Ebene, auf der die eigentlichen Kernprozesse stattfinden und die Kernkompetenzen vorhanden sind, die notwendige Akzeptanz fehlt. Problembewusstsein und Sicherheitskultur können schlicht nicht verordnet werden, sondern müssen sich von innen heraus entwickeln - auch wenn es dafür klare Strukturen und Leitplanken braucht, die von der Führung vorgegeben werden und deren Berücksichtigung eingefordert wird.
Ein weiteres Problem ergibt sich aus der Verschiedenheit der Bewertungskriterien und der Ableitung der daraus resultierenden Konsequenzen. Fast immer beruhen Bewertungen von Risiken zu weiten Teilen auf subjektiven oder nur semi-quantitativen Einschätzungen und können folglich stark variieren. Gerade für die Bewertung von klinischen Risiken mangelt es oft an validen, vollständigen und repräsentativen Daten, die hierbei zu Hilfe genommen werden könnten. Häufig werden deshalb Abschätzungen z.B. auf Basis von Haftungsansprüchen (Schadenszahlen und -höhe) verwendet. Dies geschieht besonders dann, wenn klinische Risiken aus der IKS-Optik heraus gewichtet werden. Jedoch ergibt sich hierbei ein verzerrtes Bild der tatsächlichen Situation. Bekanntermassen sind hier invasive Eingriffe und die Geburtshilfe stark überrepräsentiert, während z.B. die deutlich häufigeren Medikationsfehler in den Schadensstatistiken aus der Haftungsoptik deutlich seltener erscheinen.

Wenn also vor allem Haftungsansprüche als Grundlage für die Ausrichtung des klinischen Risikomanagements dienen, würde der Fokus hauptsächlich auf die Vermeidung von Ereignissen gerichtet, die zu Schadensersatzzahlungen führen (bzw. bereits geführt haben). Aus Sicht des «unternehmerischen Risikos» ist dies zwar nachvollziehbar, da Haftungsansprüche hierfür die relevante Grösse sind. Man stützt sich dabei aber auf eine Risikoeinschätzung, die der tatsächlichen klinischen Risikosituation überhaupt nicht entspricht. Aus Sicht der Patientensicherheit ist es deshalb nicht vertretbar, dass Ressourcen nur auf Risiken konzentriert werden, die in seltenen Fällen zu hohen Schadenersatzzahlungen führen, hingegen Risiken, die bei vielen Patienten zu Schädigungen führen und keine Zahlungen auslösen, weniger beachtet werden. Klar wird dabei, 
dass sich die Bewertungslogiken zwischen klinischem und Gesamtrisikomanagement systematisch und substantiell unterscheiden können.

Es ist grundsätzlich nicht falsch, wenn Spitäler versuchen, die Kosten, die sich durch klinische Risiken ergeben können, nach Möglichkeit zu reduzieren. Im Gegenteil, angesichts knapper werdender Ressourcen ist es sogar die Pflicht einer Spitalleitung, vermeidbare Ausgaben zu begrenzen. Allerdings dürfen aber Massnahmen zur Verbesserung der Patientensicherheit nicht primär von einem günstigen Kosten-Nutzen-Verhältnis abhängig gemacht werden. Der Schutz der Patienten ist eine grundsätzliche Pflicht eines Spitals, die Vorrang vor wirtschaftlichen Überlegungen haben muss. Risiko-Bewertungen auf rein ökonomischer Basis sind deshalb abzulehnen. Allein schon der Eindruck, Spitäler würden auf diese Weise kalkulieren, hätte in der öffentlichen Wahrnehmung eine verheerende Auswirkung.

Diese Überlegungen zeigen, wie wichtig es ist, Patientensicherheit nicht einfach in die Reihe der allgemeinen Risikomanagement-Massnahmen und -Methoden einzuordnen. Die Ziele mögen sich zwar zum Teil überschneiden, der grundlegende Unterschied bleibt jedoch bestehen. Klinisches Risikomanagement dient in erster Linie dem Schutz der Patienten vor Gefahren, die von der Behandlung im Spital ausgehen. Die allgemeinen Risikomanagement-Massnahmen sollen hingegen die Gefahren für das Unternehmen Spital abwehren.

\section{Abbildung 2}

Klinisches Risikomanagement ist eigentlich das, was in anderen Branchen (z. B. in der Flugsicherheit) als SafetyManagement bezeichnet wird.

\section{Literatur}

1 Middendorf C. Aufgaben, Inhalte und Ansatzpunkte des Risikomanagements. In: von Eiff W (Hrsg.). Risikomanagement. Kosten-/Nutzenbasierte Entscheidungen im Krankenhaus. Wegscheid: WIKOM; 2006.

2 Hart D. Patientensicherheit, Fehlermanagement, Arzthaftungsrecht, zugleich ein Beitrag zur rechtlichen Bedeutung von Empfehlungen. Medizinrecht. 2012;1:1ff.

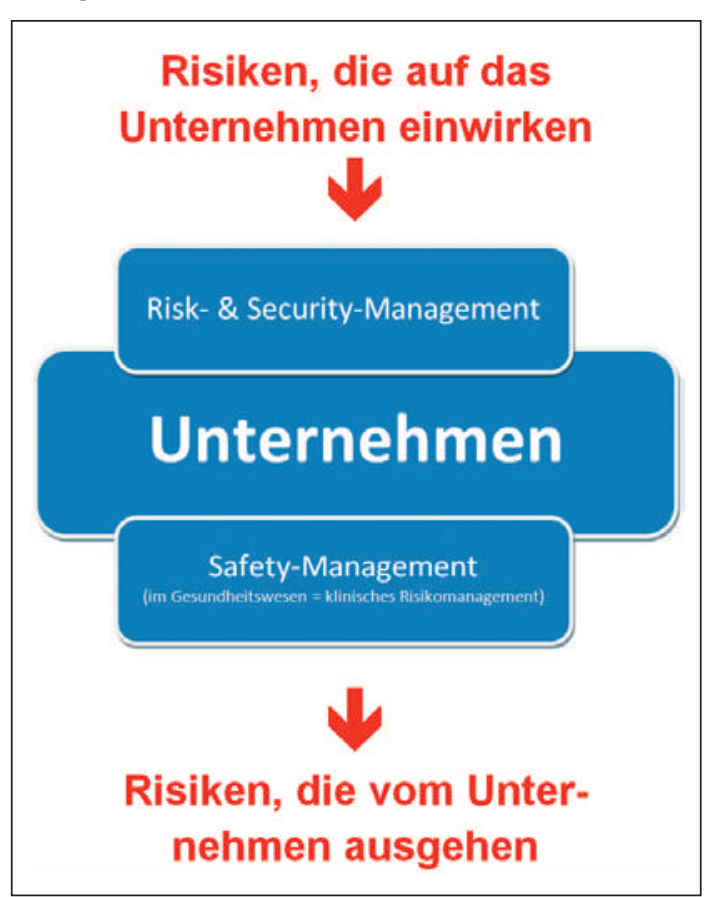

Trotz der unterschiedlichen Zielsetzungen zeigen sich immer wieder Probleme, die auf eine fehlende Differenzierung und Vermischung zwischen diesen Aufgabenbereichen zurückzuführen sind. Hier trägt sicherlich auch die gemeinsame Verwendung des Oberbegriffes Risikomanagement $\mathrm{zu}$ einer Verwirrung bei. In anderen Branchen werden die verschiedenen Zielrichtungen durch differenziertere Begrifflichkeiten besser voneinander abgegrenzt. Meist wird dabei zwischen RisikoManagement, Security-Management und SafetyManagement unterschieden. Während das RisikoManagement die Unternehmensrisiken abdeckt, dient das Security-Management dem Schutz vor Delikten der verschiedensten Art (z.B. Diebstahl, Cyber-Kriminalität). Aufgabe des sogenannten Safety-Managements ist es hingegen, die Kernprozesse eines Unternehmens und damit seine Produkte und Dienstleistungen möglichst sicher zu gestalten, um die anvertraute Klientel vor Schäden zu schützen (Abb.2). Klinisches Risikomanagement ist also eigentlich das, was in anderen Branchen (z.B. in der Flugsicherheit) als Safety-Management bezeichnet wird.

Da im deutschsprachigen Raum die Begriffe «Patientensicherheit» und «klinisches Risikomanagement» seit langem miteinander verknüpft sind, wären Hoffnungen auf eine nachträgliche begriffliche Differenzierung wohl illusorisch. Allerdings ist in der Praxis darauf zu achten, dass die Kerngedanken der Patientensicherheit erhalten bleiben und hinsichtlich der Zielrichtung und Bewertung die unternehmerischen, ökonomischen oder juristischen Überlegungen nicht überhandnehmen. Das klinische Risikomanagement muss nahe an den klinischen Prozessen bleiben, nämlich dort, wo Risiken für Patienten entstehen, um Stärke und Verbindlichkeit aufzubauen und um attraktiv für die klinischen Fachpersonen zu werden. Dazu müssen alle Erkenntnismittel für Risiken und Fehler wie Fehlermeldesysteme, Schadensanalysen, Beschwerdemanagement, Chart Review-Verfahren und Patienten- sowie Mitarbeiterrückmeldungen systematisch genutzt werden. Auf dieser Basis ist es auch möglich, das klinische Risikomanagement als einen essentiellen Teil des spitalweiten Gesamt-Risikomanagementsystems zu betreiben, ohne dass dadurch die Sicherheit für den individuellen Patienten vernachlässigt würde.

Entscheidend dabei ist, dass die Verantwortungsträger in Gesundheitsbetrieben die Unterschiede zwischen gesamtbetrieblichem Risikomanagement (IKS) und klinischem Risikomanagement sowie die methodischen, organisatorischen und inhaltlichen Anforderungen an das klinische Risikomanagement bewusst berücksichtigen und zur Führungsaufgabe erklären und ihm so das erforderliche Gewicht in der Organisation geben, deren Kernaufgabe ( Core-business») die Betreuung und Behandlung von Patientinnen und Patienten ist. 